\title{
Relação entre escores de desempenho motor e aptidão física em crianças com idades entre 07 e 08 anos
}

\section{Relationship between motor performance and physical fitness score in 7- to 8-year-old children}

\author{
Ruy Jornada Krebs ${ }^{1,2}$ \\ Marcelo Gonçalves Duarte \\ Glauber Carvalho Nobre \\ Patrik Felipe Nazario \\ João Otacílio Libardoni dos Santos
}

\section{Universidade do Estado de Santa Catarina - Cen tro de Ciências da Saúde e do Esporte. Programa de Pós-Graduação em Ciências do Movimento Humano. Laboratório de Desenvolvimento e Aprendizagem Motora. Santa Catarina. Brasil. \\ 2 "in memorian".}

Enviado em 23/09/10 Revisado em 17/10/10 Aprovado em 10/11/10
Resumo - Existem muitas sugestões de testes que se propõem a avaliar o desempenho motor e a aptidão física. Estes testes levam em consideração diferentes critérios para análise do desempenho como formas e padrões de movimento, tempo, distância e repetições em cada tarefa. $\bigcirc$ objetivo deste estudo foi investigar a relação entre os escores de desempenho motor e aptidão física de crianças. Participaram deste estudo, 50 crianças (25 meninos e 25 meninas) com idades entre 7 e 8 anos e de ambos os sexos. O Test of Gross Motor Development - Segunda Edição (TGMD-2) foi utilizado para avaliar o desempenho motor. Para avaliação da aptidão física, foram utilizados 3 testes específicos propostos pela American Alliance of Health, Physical Education and Recreation (AAHPERD). Os resultados mostraram diferenças estatísticas e correlações fracas em algumas habilidades como corrida, salto horizontal, saltito, passada, arremesso por baixo e arremesso de medicine ball. $\mathrm{O}$ uso equivalente de ambos os testes pode não ser adequado, visto que cada teste tem suas próprias peculiaridades a respeito dos critérios de avaliação.

Palavras-chave: Aptidão física; Desempenho motor; Crianças; Educação física.

Abstract - There are many tests for the evaluation of motor performance or physical fitness that take into account different criteria such as movement forms and patterns, time, distance, and repetitions of each task for the analysis of performance. The aim of this study was to investigate the relationship between motor performance and physical fitness scores in children. Fifty children of both genders aged 7 to 8 years ( 25 boys and 25 girls) participated in this study. The Test of Gross Motor Development - Second Edition (TGMD-2) was used for the evaluation of motor performance. Physical fitness was assessed by three specific tests proposed by the American Alliance for Health, Physical Education, Recreation and Dance (AAHPERD). The results showed statistical significance and weak correlations among some skills such as run, horizontal jump, hop, leap, underhand roll, and medicine ball throw. The equivalent use of both tests may not be adequate since each test has its own peculiarities in terms of assessment criteria.

Key words: Physical fitness; Motor performance; Children; Physical education. 


\section{INTRODUÇÃO}

A avaliação do desempenho motor em crianças e adolescentes, mesmo como medida monocasional, fornece informações relevantes para o planejamento de programas de educação física e esportes, na formulação de estratégias de intervenção motora, na programação de rotinas de atividades e exercícios físicos, entre outros ${ }^{1,2}$. Dessa forma, tem-se observado diversas sugestões de baterias de testes para avaliar o desempenho motor, tais como: Körperkoordination Test für Kinder (KTK) proposta por Kiphard e Schiling ${ }^{3}$; Bruininks-Oseretsky Test of Motor Proficiency (BOTMP), proposta por Bruininks; ${ }^{4}$ Movement Assessment Battery for Children (MABC), proposta por Henderson e Sugden ${ }^{5}$; Peabody Developmental Motor Scales - second edition (PDMS-2), proposta por Folio e Fewell ${ }^{6}$ e o Test of Gross Motor Development-Second-Edition, proposto por Ulrich?

Igualmente ao desempenho motor, a aptidão física de crianças e adolescentes têm sido monitoradas ao longo do último século ${ }^{8,9}$, pois se sabe que existe uma relação importante entre a melhora da aptidão física e a melhora nas capacidades funcionais motoras (força, velocidade, agilidade, flexibilidade e potência aeróbia) dos indivíduos, contribuindo, assim, na eficiência da realização de determinadas tarefas. Nessa perspectiva, diversas baterias de testes estão sendo utilizadas por estudiosos neste tipo de avaliação, dentre as quais se destacam: Physical Best proposta pela American Alliance of Health, Physical Educantion and Recreation (AAHPERD) que é uma das baterias de testes para avaliação da aptidão física que têm recebido maior aceitação entre os pesquisadores nos Estados Unidos ${ }^{10}$; Eurofit a qual tem recebido maior destaque no continente Europeu, e vem sendo utilizada em diversos estudos ${ }^{11,12} \mathrm{e}$ no Brasil, uma bateria de testes semelhante tanto à bateria da AAHPERD quanto a do Eurofit, nomeada de Projeto Esporte Brasil ${ }^{13}$.

Ao observar as diferentes baterias de testes e as características específicas de cada uma delas, é possível constatar que as baterias criadas para avaliar o desempenho motor focam-se em análises qualitativas do movimento, nas quais se evidencia uma observação sistemática com julgamento criterioso da qualidade e da forma do movimento (técnica de realizar os movimentos específicos em termos de seus componentes, rotação dos quadris, ação do braço, ação da perna, etc). Já ao verificar os testes propostos para avaliar a aptidão física, esses apontam escores de ações motoras em termos quantitativos (tempo de duração de corrida, distância que uma criança consegue saltar, velocidade de movimento, número de repetições). Até o presente momento, poucos estudos ${ }^{14-16}$ procuraram observar a relação da aptidão física e do desempenho motor (relação entre as variáveis qualitativas e quantitativas). Dessa forma, o objetivo do presente estudo foi investigar a relação entre os escores obtidos em testes que avaliam o desempenho motor (avaliado de forma qualitativa) e aptidão física (avaliado de forma quantitativa) de crianças eutróficas entre 7 e 8 anos de idade.

\section{PROCEDIMENTOS METODOLÓGICOS}

$\mathrm{O}$ delineamento do presente estudo inclui a relação dos escores obtidos das varáveis desempenho motor e aptidão física de crianças eutróficas entre 7 e 8 anos de idade. A pesquisa foi aprovada pelo Comitê de Ética em Pesquisa em Seres Humanos CEP da Universidade do Estado de Santa Catarina - UDESC (protocolo no 26/2009). Inicialmente, foi feito contato com a direção da escola para esclarecimentos sobre os procedimentos a serem realizados e o objetivo do estudo, e também, com os pais ou responsáveis pelas crianças, pois sendo os participantes da pesquisa menores de 18 anos foi necessária a assinatura do Termo de Consentimento Livre e Esclarecido, bem como a permissão para a filmagem. Para a seleção dos participantes da pesquisa, determinou-se como critério de inclusão que as crianças apresentassem um estado nutricional de eutrofia, conforme a Organização Mundial da Saúde ${ }^{17}$. Assim, participaram deste estudo 50 crianças de ambos os sexos, das quais foram sorteadas 25 crianças do sexo masculino e 25 crianças do sexo feminino, oriundas de uma escola da rede pública de ensino do município de Florianópolis/ SC, a qual reúne crianças de diversos estratos sociais e residentes em diferentes regiões da cidade.

As variáveis selecionadas para o estudo foram Desempenho Motor e Aptidão Física. Em relação ao Desempenho Motor, avaliaram-se as variáveis Habilidades de Locomoção (Correr, Galopar, Salto com 1 Pé, Passada, Salto Horizontal, Corrida) e as Habilidades de Controle de Objetos (Lateral, Rebater, Quicar, Receber, Chutar, Arremesso por Cima e Arremesso por Baixo). Já na aptidão Física, avaliaram-se as variáveis Velocidade de 20 Metros, Salto Horizontal e Arremesso de Medicine Ball.

Para a aquisição dos dados relacionados ao desempenho motor, foi utilizado o Test of Gross Motor Development - Second Edition (TGMD-2) 
desenvolvido por Ulrich, validado para uma população de crianças com idade entre 3 e 10 anos, provenientes da região sul do Brasi ${ }^{18}$. Cada criança foi avaliada individualmente, em um tempo médio de 20 minutos, onde essas foram orientadas por somente um pesquisador. Utilizou-se uma câmera filmadora, posicionada frontalmente, conforme protocolo estabelecido pelo autor do teste, e uma câmera lateral para registro e posterior análise do desempenho motor dos participantes ${ }^{19}$. Três pesquisadores vinculados ao Laboratório de Desenvolvimento e Aprendizagem Motora - LADAP do Centro de Ciências da Saúde e do Esporte CEFID - UDESC realizaram as análises dos vídeos ${ }^{18}$. Para avaliação da aptidão física utilizou-se os testes específicos de força de membros inferiores (Salto Horizontal), velocidade de deslocamento (Velocidade de 20 metros) e força de membros superiores (Medicine Ball) proposta pela American Alliance of Health, Physical Educantion and Recreation ${ }^{10}$ para indivíduos com idade entre 7 a 17 anos. Estes testes foram realizados no mesmo local onde as crianças realizaram os testes de desempenho motor. Pretendendo minimizar os efeitos de possível fadiga fisiológica, seguiu-se uma ordem sequencial da realização dos testes: 1 - Salto Horizontal, 2 - Corrida em 20 Metros e 3 - Arremesso de Medicine Ball, bem como a aplicação destes, no mínimo, nos dois dias posteriores à realização dos testes de desempenho motor. As crianças foram familiarizadas com os testes e tiveram um período de experimentação e explicação. Após demonstração realizada pelo avaliador, dois ensaios foram efetuados pelos participantes, visando uma correta execução, sendo escolhido o melhor resultado de cada teste. Os três testes empregados neste estudo seguiram o protocolo sugerido pela AAHPERD ${ }^{10}$. Entretanto, na mensuração do tempo de deslocamento, foi substituído o cronômetro por dois pares de fotocélulas dispostas a uma distância de 20 metros entre si.

Para a análise dos dados, foram utilizados os seguintes procedimentos: a) foi realizado o teste de normalidade de Shapiro-Wilk e observou-se que os dados não seguiam uma distribuição normal ( $p<0,001)$ optando-se, desta forma, por realizar, posteriormente, análises estatísticas não paramétricas, b) os escores obtidos nos testes de desempenho motor e aptidão física foram descritos por meio de mediana, valor máximo e mínino, c) para melhor descrição dos resultados, utilizou-se, também, a análise do percentual de sujeitos que se encontravam abaixo, dentro e acima da mediana dos escores relativos ao desempenho de cada habilidade motora, bem como o escore máximo, possível de ser alcançado em cada habilidade do teste d) os escores obtidos nos testes de desempenho motor e os escores obtidos nos testes de aptidão física foram convertidos em valores de "Z" (para se obter escalas de medidas em níveis idênticos) e) para verificar possíveis relações entre as variáveis, posteriormente, utilizou-se o teste de correlação de Kendau Taus, , analisando-se somente as variáveis que apresentavam padrões motores similares em ambas as baterias de teste. $\mathrm{O}$ nível de significância adotado foi de $\mathrm{p} \leq 0,05$.

\section{RESULTADOS}

Na Tabela 1, são apresentados os resultados dos testes de Desempenho Motor (Locomoção e Controle de Objetos) de cada habilidade, no qual, ao observar as habilidades de Locomoção (Correr, Galopar, Salto com 1 Pé, Passada, Salto Horizontal, Corrida Lateral), destaca-se que os escores brutos mais elevados do grupo de estudo foram obtidos na Corrida e na Passada, e tanto o valor máximo quanto o valor da mediana foram iguais ao valor máximo possível de ser alcançado no teste (melhor desempenho possível). Nestas habilidades, a grande maioria das crianças apresentou desempenho considerado como dentro da mediana. Nas habilidades de Salto com um Pé, Salto Horizontal e Galopar foram constados os maiores percentuais acima da mediana de desempenho. A Corrida Lateral foi a habilidade motora investigada que indicou maiores valores percentuais de crianças abaixo da mediana de desempenho.

Ao analisar isoladamente as habilidades de Controle de Objeto (Tabela 01), pode-se observar que, na tarefa de Chutar, os participantes obtiveram o melhor desempenho, e também o valor da mediana foi igual ao valor máximo a ser alcançado no teste. O Rebater e o Quicar foram as habilidades motoras investigadas que indicaram maiores valores percentuais de crianças abaixo da mediana de desempenho.

A Tabela 2 apresenta os escores dos testes de Aptidão Física, na qual se observa que no teste de Velocidade de 20 Metros, somente 12\% dos participantes estiveram abaixo da mediana, e $40 \%$ encontravam-se acima da mediana. No Salto Horizontal, 46\% das crianças investigadas ficaram abaixo da mediana, e somente $4 \%$ na mediana. Quando se analisou os escores obtidos no teste de Arremesso de Medicine Ball observou-se que 48\% dos participantes ficaram abaixo da mediana.

Na Tabela 3, estão descritos os coeficientes de correlação (r) obtidos entre os escores dos testes 
Tabela 1. Escores brutos mínimos, máximos, mediana e percentual dos participantes em cada habilidade do TGMD-2.

\begin{tabular}{lccccccc}
\hline Habilidades & $\mathrm{E}_{\text {mat }}$ & $\mathrm{E}_{\text {miat }}$ & $\mathrm{E}_{\text {maat }}$ & Mediana & $\begin{array}{c}\text { \% Abaixo } \\
\text { Mediana }\end{array}$ & \% Mediana & $\begin{array}{c}\text { \% Acima } \\
\text { Mediana }\end{array}$ \\
\hline Correr & 8 & 6 & 8 & 8 & 2 & 98 & 0 \\
Galopar & 8 & 2 & 8 & 6 & 22 & 44 & 34 \\
Salto com 1 pé & 10 & 0 & 10 & 8 & 26 & 30 & 44 \\
Passada & 6 & 5 & 6 & 6 & 2 & 98 & 0 \\
Salto Horizontal & 8 & 3 & 8 & 6 & 12 & 52 & 36 \\
Corrida Lateral & 8 & 4 & 8 & 7 & 48 & 30 & 22 \\
Rebater & 10 & 2 & 10 & 9 & 46 & 24 & 30 \\
Quicar & 8 & 0 & 8 & 7 & 46 & 6 & 48 \\
Receber & 6 & 0 & 6 & 4 & 40 & 26 & 34 \\
Chutar & 8 & 7 & 8 & 8 & 12 & 88 & 0 \\
Arremesso por cima & 8 & 2 & 8 & 6 & 34 & 34 & 32 \\
Arremesso por baixo & 8 & 2 & 8 & 6 & 32 & 46 & 22 \\
\hline
\end{tabular}

* $\mathrm{E}_{\text {mat }}$ : máximo de pontos possível de ser atingido no TGMD-2

** $\mathrm{E}_{\text {miat }}:$ mínimo de pontos atingido pelos participantes nas habilidades

*** $\mathrm{E}_{\text {maat }}$ : máximo de pontos atingido pelos participantes nas habilidades

Tabela 2. Escores mínimos, máximos, mediana e percentual dos participantes em cada teste de Aptidão Física.

\begin{tabular}{lcccccc}
\hline Habilidades & $\mathrm{E}_{\text {miat }}{ }^{*}$ & $\mathrm{E}_{\text {maat }}$ & Mediana & $\begin{array}{c}\text { \% Abaixo } \\
\text { Mediana }\end{array}$ & \% Mediana & $\begin{array}{c}\text { \% Acima } \\
\text { Mediana }\end{array}$ \\
\hline Velocidade de 20 metros $(\mathrm{s})$ & 3,17 & 4,51 & 3,75 & 12 & 48 & 40 \\
Salto Horizontal $(\mathrm{cm})$ & 100 & 166 & 135 & 46 & 4 & 50 \\
Arremesso Medicine Ball $(\mathrm{cm})$ & 111 & 213 & 172 & 48 & 2 & 50 \\
\hline
\end{tabular}

* $\mathrm{E}_{\text {miat }}$ : mínimo de pontos atingido pelos participantes nas habilidades;

** $\mathrm{E}_{\text {maat }}$ : máximo de pontos atingido pelos participantes nas habilidades.

Tabela 3. Valores das correlações (r) entre os escores de desempenho motor e aptidão física dos participantes do estudo.

\begin{tabular}{|c|c|c|c|}
\hline & \multicolumn{3}{|c|}{ Aptidão Física } \\
\hline $\begin{array}{l}\text { Desempenho Motor } \\
\text { (TGMD-2) }\end{array}$ & $\begin{array}{l}\text { Velocidade de } \\
20 \text { metros }\end{array}$ & Salto Horizontal & Arremesso Medicine Ball \\
\hline Correr & $r=0,209^{*}$ & $r=-0,259^{*}$ & - \\
\hline Galopar & $r=0,010$ & $r=0,078$ & - \\
\hline Saltar com 1 pé & $r=0,117$ & $r=-0,263^{*}$ & - \\
\hline Passada & $r=0,099$ & $r=-0,207^{*}$ & - \\
\hline Salto Horizontal & $r=0,028$ & $r=0,070$ & - \\
\hline Corrida Lateral & $r=0,079$ & $r=-0,033$ & - \\
\hline Rebater & - & - & $r=0,069$ \\
\hline Quicar & - & - & $r=0,113$ \\
\hline Receber & - & - & $r=0,097$ \\
\hline Chutar & - & - & $r=0,000$ \\
\hline Arremesso por Cima & - & - & $r=0,065$ \\
\hline Arremesso por Baixo & - & - & $r=0,239^{*}$ \\
\hline
\end{tabular}

$\left({ }^{*}\right)$ Correlações significativas $p \leq 0,05$.

de Desempenho Motor (TGMD-2) e de Aptidão Física (AAHPERD). Pode-se perceber uma correlação fraca $(r=0,209)$ entre o escore do Correr (TGMD-2) e o escore da Corrida de 20 metros da Aptidão Física. Nota-se, também, que no escore do teste Salto Horizontal da Aptidão Física houve uma correlação fraca com o Correr, Saltar com um Pé e a Passada (TGMD-2), porém essas correlações todas negativas $(\mathrm{r}=-0,259, \mathrm{r}=-0,263, \mathrm{r}=-0,207$ respectivamente). Ao analisar o teste de Arremesso por Baixo (TGMD-2) e o teste do Arremesso de Medicine Ball da Aptidão Física, também se obteve uma correlação fraca $(r=0,239)$.

\section{DISCUSSÃO}

Observando os escores de desempenho motor acima (Tabela 01), no geral, verifica-se que a maioria dos 
participantes deste estudo encontra-se na mediana ou acima da mediana nos níveis de desempenho das habilidades de locomoção e de controle de objetos, demonstrando padrões satisfatórios de movimento. Estes resultados confrontam os achados de diversos estudos $^{2,14,20-22}$ encontrados na literatura, pois nesses estudos os autores observaram escores inferiores ao do presente estudo. Uma possível explicação é que todos os participantes deste estudo apresentavam um estado nutricional de eutrofia, e também se tratavam de crianças que deveriam estar no final da fase do movimento fundamental (estágio maduro) e iniciando a fase especializada das habilidades motoras, mais especificadamente, no estágio de transição.

Ao destacar o desempenho das habilidades motoras separadamente, resultados semelhantes aos deste estudo foram achados por Goodway, et al..$^{23}$ em relação à habilidade de Saltar com um Pé, onde essa habilidade apresentou a maior média de desempenho. No entanto, estes autores identificaram que as crianças obtiveram uma média de escore alto na habilidade de galopar, diferentemente deste estudo realizado. Contudo, no estudo de Braga et al. ${ }^{24}$, com crianças de 6 e 7 anos de idade, encontraram-se resultados inferiores aos escores das habilidades de locomoção (Correr, Galopar, Salto com 1 Pé, Passada, Salto Horizontal, Corrida Lateral), achados no presente estudo. $O$ mesmo foi observado quando se analisou o estudo de Brauner e Valentini ${ }^{22}$ com crianças de 5 e 6 anos de idade, onde os escores brutos encontrados das habilidades do TGMD. 2 também foram inferiores ao presente estudo. Unicamente, o escore da habilidade de receber foi semelhante a este estudo, sugerindo que esta habilidade pode alcançar o estágio maduro mais rapidamente que as outras. Estas diferenças estão também relacionadas às idades das amostras dos estudos, visto que as crianças desse estudo devem estar no final da fase do movimento fundamental.

Ao relacionar o desempenho motor com variáveis da aptidão física neste estudo observaram-se correlações fracas e somente entre algumas habilidades (Correr TGMD-2 e Corrida de 20 metros; Salto Horizontal da Aptidão Física e Correr TGMD-2; Salto Horizontal da Aptidão Física e saltar com um Pé e a Passada TGMD-2; Arremesso por Baixo do TGMD-2 e Arremesso de Medicine Ball da Aptidão Física) (Tabela 2). Isso demonstra que as formas de avaliação quantitativas e qualitativas não possuem equivalências para as variáveis analisadas neste estudo, fazendo com que seja utilizada uma ou outra forma de avaliação. Portanto, estes achados vão de encontro ao estudo realizado por Haga ${ }^{15}$, no qual a autora investigou a relação entre aptidão física e competência motora de 67 crianças de 9 a 10 anos, utilizando a bateria de testes $\mathrm{MABC}$ e 09 testes específicos de aptidão física, e ressaltou em seus resultados que tanto para a contagem geral ou para as três subcategorias do $M A B C$, existia uma correlação forte com os escores da aptidão física (encontrou equivalência entres as variáveis qualitativas e quantitativas) para ambos os sexos dentro da respectiva amostra. Ainda, em outro estudo ${ }^{16}$, utilizando variáveis quantitativas e qualitativas, a autora comparou a aptidão física e competência motora de crianças e também encontrou associação entre as variáveis.

A inexistência de correlações significativas moderadas e fortes neste estudo e as divergências encontradas com a literatura podem estar relacionadas ao fato de que as exigências nas tarefas analisadas são diferentes. Por exemplo, as habilidades de salto com um pé e a passada podem requerer mais coordenação motora e equilíbrio, enquanto que o teste de salto horizontal, como medida de aptidão física, exige primordialmente mais força/potência de membros inferiores. Outra questão relevante é que a forma como cada teste analisa a eficiência do movimento, e classifica em níveis adequados ou não, são diferentes, pois a mesma criança pode obter um padrão satisfatório de movimento na corrida (sincronia na movimentação dos braços, tronco ereto, etc.) quando avaliado pelo TGMD-2, mas um desempenho insatisfatório no tempo do teste de velocidade de 20 metros, pois mesmo apresentando o padrão adequado não consegue obter uma boa velocidade. Entende-se, também, que crianças com melhores escores de aptidão física, no salto horizontal, por exemplo, não necessariamente obteriam escores análogos no teste de desempenho motor proposto pelo TGMD-2. Ressalta-se, ainda, que existem limitações neste estudo e que necessitam ser consideradas em estudos futuros, como por exemplo, aspectos relacionados à maturação biológica, aos hábitos de vida e ao histórico do envolvimento em práticas esportivas, as quais podem influenciar o desempenho e a aptidão física das crianças.

\section{CONCLUSÃO}

Após relacionar o desempenho motor, analisado qualitativamente, e aptidão física, analisada quantitativamente, em crianças eutróficas com idade entre 07 e 08 anos, pode-se concluir que, devido aos testes utilizados apresentarem particularidades quanto à proposta de avaliação (diferentes crité- 
rios de análise para o desempenho considerado satisfatório), o uso equivalente desses testes pode ser inadequado. Fica evidente, também, que essas baterias de teste devem ser utilizadas conforme o objetivo e as fases do planejamento dos programas estabelecidos pelo avaliador para a mensuração do desempenho das crianças e dos adolescentes. Dessa forma, enfatiza-se que sejam utilizadas, inicialmente, as baterias para avaliação da qualidade do movimento, a qual é um parâmetro importante para verificar o processo de aprendizagem e, consequentemente, após a criança ter alcançado autonomia para realizar a tarefa motora, enfatizar os testes de aptidão física.

\section{REFERÊNCIAS BIBLIOGRÁFICAS}

1. Guedes DP, Guedes JERP, Barbosa DS, Oliveira JA. Atividade Física Habitual e Aptidão Física Relacionada à Saúde em Adolescentes. Rev Bras Ciên Mov 2002;10(1):1-21.

2. Surdi AC, Krebs RJ. Estudo dos padrões fundamentais de movimento de pré escolares que participaram do programa de desenvolvimento infantil do SESI da cidade de Videira SC. Kinesis 1999;1(21):57-69.

3. Kiphard EJ, Shilling VF. Körper-koodinations-test für kinder KTK. Manual Von Fridhelm Schilling. Wenhein: Beltz Test; 1974.

4. Bruininks RH. Bruininks-Oseretsky Test of Motor Proficiency Examiners Manual. Circle Pines, MN: American Guidance Service; 1978.

5. Henderson SE, Sugden DA. Movement Assessment Battery for Children. Sidcup, UK: Therapy Skill Builders; 1992.

6. Folio R; Fewwel, R. Peabody Develomental Motor Scales-2. San Antonio: The Psychological Corporation; 2000.

7. Ulrich DA. Test of gross motor development - second edition: examiner's manual. Austin, Texas: Pro. Ed; 2000.

8. Reiff G, Dixon W, Jocaby D, Ye G, Spain C, Huniscker P. The President's Council on Physical Fitness and Sports national school population fitness survey: Ann Arbor, University of Michigan; 1986.

9. Simons J, Beunen GP, Renson R, Claessens ALM, Vanreusel B, Lefevre JAV. Growth and fitness of Flemish girls. The Leuven Growth study. Champaing: Human Kinetics Publishers; 1990.

10. American Alliance for Health, Physical Educantion and Recreation and Dance/ AAHPERD. Physical Best: Reston Virgínia; 1988.

11. Volbekiene V, Griciltte A. Health-related physical fitness among schoolchildren in Lithuania: A comparison from 1992 to 2002. Scand J Public Health, 2007;35(3): 235-42

12. Skowronski W. Horvat M, Nocera J, Roswal G, Croce R. Eurofit Special: European Fitness Battery Score Variation Among Individuals With Intellectual Disabilities. Adapt Phys Activ Q 2009;26(1):54-67
13. Projeto Esporte Brasil/ Proesp-Br. Ministério dos Esportes, 2007. Disponivel em: < http://www.esef.ufrgs. br/proesp-br/proespbr.htm $>$ [2010 set 10].

14. Copetti FR. Nível de maturação dos padrões fundamentais de movimentos e o desempenho motor em pré-escolares. Cinergis 2000;1(1):39-50.

15. Haga $M$. The relationship between physical fitness and motor competence in children. Child Care Health Dev 2008;34(3):329-34.

16. Haga PT. Physical fitness in children with high motor competence is different from that in children with low motor competence. Phys Ther 2009;89(19):1089-97.

17. World Health Organization/WHO. Child Growth Standards: Length/height-for-age, weight-forage,weight-for-length, weight-for-height and body mass index-for-age. Methods and development. (nonserial publication). Geneva, Switzerland: 2006. Available from: http://www.who.int/childgrowth/en/ [2009 jul 10].

18. Valentini NC, Barbosa MLL, Cini GW, Pick RK, Spessato BC, Balbinoti MA. Teste de desenvolvimento motor grosso: validade e consistência interna para uma população gaúcha. Rev. Bras. Cineantropom. Desempenho Hum 2008;10(4):399-404.

19. Ulrich, DA. The Test of Gross Motor Development-2nd Edition: Uses, Administration, and Applications. Rev Socied Bras Ativ Motora Adaptada 2005; 10(1):13-15

20. Crippa LR, Souza JM, Simoni S, Rocca RD. Desempenho motor de crianças obesas: uma investigação do processo e produto de habilidades motoras fundamentais. Rev Educ Fís/UEM 2003;14(2):13-20.

21. Valentini NC. A influência de uma intervenção motora no desempenho motor e na percepção de competência de crianças com atrasos motores. Rev paul Educ Fís 2002;16(1):61-75

22. Brauner LM, Valentini NC. Análise do desempenho motor de crianças participantes de um Programa de atividades físicas. Rev Educ Fís/UEM 2009;20(2):205-216

23. Goodway JD, Crowe H, Ward P. Effects of motor skill instruction on fundamental motor skill development. Adapt Phys Activ Q 2003; 20(1):291-314.

24. Braga RK, Krebs RJ, Valentini NC, Tkac CM. Influência da intervenção no desempenho motor das habilidades locomotoras em crianças entre 6 e 7 anos de idade. Rev Educ Fís/UEM 2009;20(2):171-81
Endereço para correspondência
João Otacilio Libardoni dos Santos
Universidade do Estado de Santa Catarina
Centro de Ciências da Saúde e do Esporte
Laboratório de Desenvolvimento e Aprendiza-
gem Motora CEFID/UDESC.
Rua Pascoal Simone, 358 - Coqueiros
CEP 88080-350 - Florianópolis, SC. Brasil
E-mail: jlibardoni@yahoo.com.br 